\title{
Collective hadronization and air showers: can LHC data solve the muon puzzle ?
}

\author{
Tanguy Pierog* \\ KIT, IKP, Karlsruhe, Germany \\ E-mail: tanguy.pierog@kit.edu
}

\section{Sebastian Baur}

Université Libre de Bruxelles, IIHE, Brussels, Belgium

E-mail: sebastian.baur@ulb.ac.be

\section{Hans Dembinski}

Max Planck Institute for Nuclear Physics, Heidelberg, Germany

E-mail: hdembins@mpi-hd.mpg.de

\section{Ralf Ulrich}

KIT, IKP, Karlsruhe, Germany

E-mail: Ralf.Ulrich@kit.edu

\section{Klaus Werner}

SUBATECH, University of Nantes - IN2P3/CNRS - IMT Atlantique, Nantes, France

E-mail: wernerasubatech.in2p3.fr

\begin{abstract}
The deficit of muons in the simulation of extensive air showers is a long standing problem and the origin of large uncertainties in the reconstruction of the mass of the high energy primary cosmic rays. Hadronic interaction models re-tuned after early LHC data have a more consistent description of the muon content among them but still disagree with data. Some aspects of collective hadronization due to the formation of a quark gluon plasma (QGP) have already been studied in the past as a possible reason for a larger production of muons under extreme conditions (rare, very central nuclear interactions), but without real success. Thus, we study collective effects in a different light: because of its different ratio of electromagnetic to hadronic energy, a QGP can contribute to solve the muon puzzle - in particular in the view of the most recent LHC data. It is demonstrated using a theoretical approach and tested in a realistic way by the modification of the EPOS model to produce a QGP also in not so extreme conditions with a possible large impact on air shower physics.
\end{abstract}

36th International Cosmic Ray Conference -ICRC2019-

July 24th - August 1st, 2019

Madison, WI, U.S.A.

* Speaker. 


\section{Introduction}

Despite all the efforts made to take into account the first results of proton-proton collisions at the LHC in hadronic interaction models used for air shower simulations, the observed number of muons, their height of production or even the depth of shower maximum are still not reproduced consistently by the models [1]. Furthermore, the differences in model predictions introduce uncertainties in the analysis of cosmic ray data, which are less than in the past but still exceed the experimental uncertainty in certain cases [2]. But before claiming for the need for "new physics", it is important to guarantee that all the QCD standard physics is properly taken into account in these models. For this, it is necessary to go beyond the simplest observables which are usually used to test them. The various LHC experiments provided a large amount of complex data to analyze and understand, in particular, the correlation between different observables are not yet fully investigated.

Among the hadronic interaction models used for air shower analysis only EPOs $[3,4,5,6]$ includes all the features needed to have a detailed description of the correlation between various observables [1]. Indeed, the core-corona approach in this model, which allows the production of a collective hadronization phase, appears to be a key element to reproduce LHC data. Before LHC, it was usually accepted that hydrodynamical phase expansion due to the formation of a quark gluon plasma (QGP), for instance, was possible only in central heavy-ion collisions. Proton-nucleus (pA) collisions were then used as a reference to probe the effect of such collective behavior in the final state with some nuclear effect at the initial state level, while proton-proton (pp) interactions are free from any nuclear effects. With the LHC operated in $\mathrm{pp}, \mathrm{pPb}$ and $\mathrm{PbPb}$ mode, it is now possible to compare high-multiplicity $\mathrm{pp}$ or $\mathrm{pPb}$ events with low-multiplicity $\mathrm{PbPb}$ events, which correspond to the same number of particles measured at mid-rapidity. Surprisingly, the very same phenomena are observed $[7,8]$ in all cases concerning the soft-particle production.

One of the most striking features observed in all systems is the long-range two-particle correlations and the evolution of the particle flow as described in [9]. In Ref. [10] it is demonstrated how these data from the CMS Collaboration can be reproduced and explained using an approach combining standard perturbative calculations for initial conditions and hydrodynamical calculations for the final state interactions.

At the same time, the recent results compiled by the WHISP working group [11] clearly indicate that the discrepancy in the muon production between simulations and data gradually increases with energy. It is a strong indication of a different hadronization than the one used in the current hadronic models $[12,13,14,15,16]$.

In this paper, we present a modified version of EPOS LHC [4] based on EPOS 3 [3] to study the consequence of the extended range of collective hadronization on air shower physics. We will demonstrate that this effect, which was attributed to heavy-ion collisions only so far, is very important to describe pp data in particular for strange particle production, and that it is potentially more important for air showers than initially thought.

In Section 2 the basic principles of EpOS and its modifications are presented. In Section 3 we present the changes implied by collective hadronization in the air shower development. Finally, in Section 4, the impact of the new features of EPOS on air shower simulations are shown and concluded in Section 5. 


\section{EPOS and Collective Hadronization}

EPOS is a minimum-bias Monte Carlo hadronic generator used for heavy-ion interactions with a long history of developments $[3,4,5,6,17]$.
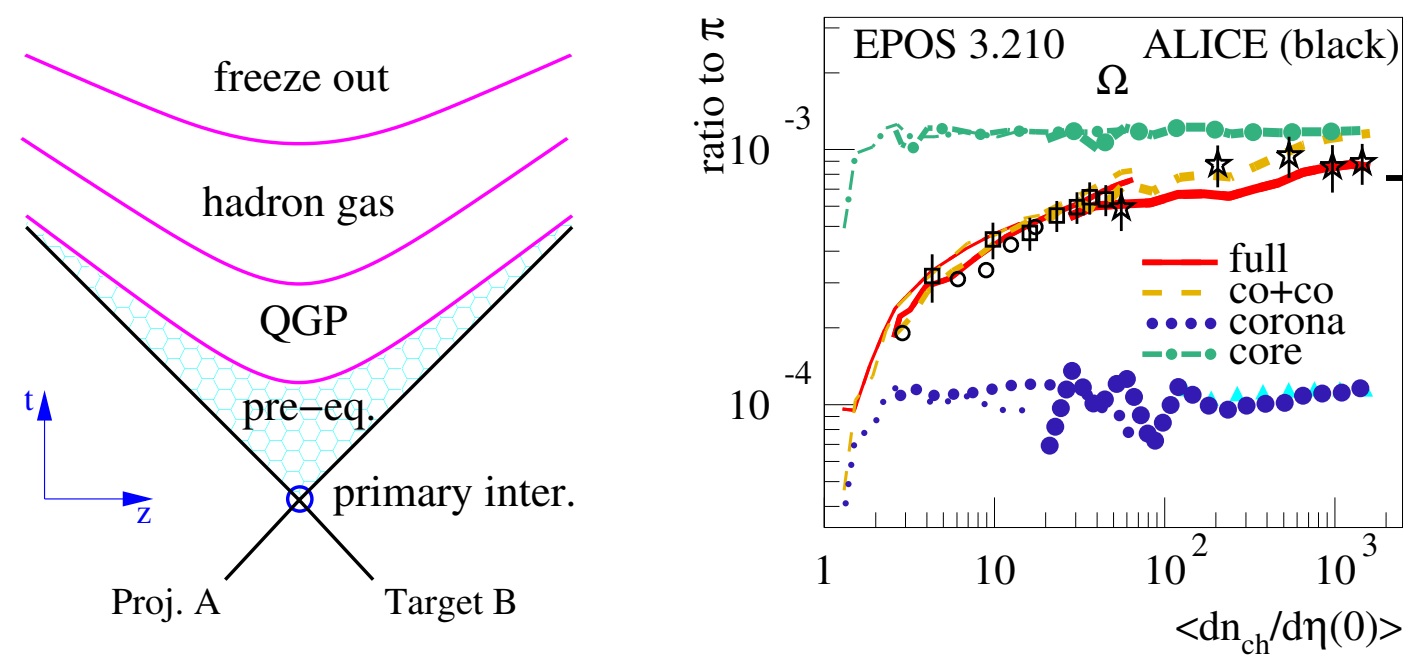

Figure 1: Left side: General space-time evolution of particles in hadronic collisions. The combination of QGP and hadron gas applies to the "core" only, and the combination of both is called collective hadronization. Right side: Particle to pion ratio for the $\Omega$ baryon versus multiplicity at mid-rapidity, for different contributions (core (dash-dotted), corona (dotted), core+corona (dashed) and all (core+corona+hadronic gas) (full)) from the EPOS simulations, for different systems (pp (thin), pPb (normal), $\mathrm{PbPb}$ (bold)). We also plot ALICE data from [7].

In Fig. 1 (left) a simplified schematic view of the space-time evolution of the high energy density part called "core" in EPOS is represented. The details of the collective hadronization phase between the primary interaction, in which the initial conditions are created, and the freeze out, after which particles travel without re-interaction, change on an event-by-event basis. The energy, impact parameter, number of multiple scatterings and geometry all change the initial conditions and the possible existence and evolution of the "core", but the same rules apply whatever energy and system are considered (from pp to heavy ions). In practice, there is no real phase boundary between the QGP and the hadron gas in this collective hadronization phase. The rest of the event with a low energy density is called "corona".

For many years, it has been well established that collective hadronization is a must to reproduce data taken with heavy ions such as gold or lead. Thanks to the measurement of various observables as a function of the particle multiplicity at LHC, it is possible to combine the different systems such as $\mathrm{pp}, \mathrm{pPb}$ and $\mathrm{PbPb}$ in a single plot and see whether different behaviors are observed. As shown in [7], no particular difference has been seen for the various systems, and there is a smooth transition from low to high multiplicity in strangeness production, for instance. Furthermore, this does not only occur at very high multiplicities reachable in $\mathrm{PbPb}$ collisions, but already starts at a quite low multiplicity with a sizable effect at the average multiplicity of pp interactions $\left(\left\langle\mathrm{d} N_{\mathrm{ch}} / \mathrm{d} \eta\right\rangle\right.$ about 6). 
As seen [7], EPOS LHC is overestimating the multiplicity of this transition. The core with collective hadronization is produced too late compared to what is observed at the LHC (and the effect was overestimated to compensate and reproduce the average correctly). An example of what can be done with EPOS 3 is visible in Fig. 1 (right). In that case, the evolution of the number of $\Omega$ baryons as a function of the multiplicity is well reproduced both in shape and amplitude. We can see that the yield ratios of the different contributions (core (dotted line) or corona (dash-dotted line)) do not depend either on the system type or on the multiplicity. The change of the ratio as a function of the multiplicity is only due to the evolution of the different fractions of particles produced by the core and the corona. On top of this, the effect of the hadron gas applies only in high-multiplicity $\mathrm{PbPb}$ events (the difference between the dashed and the full line) [18].

Since EPOS 3 is not available for air shower simulations, EPOS LHC can be adjusted to mimic the core evolution as a function of multiplicity. The hadronization scheme is changed from microcanonical to grand-canonical ensemble to be closer to the results observed in $\mathrm{PbPb}$ collisions. Since the energy density at which the core is formed has been reduced, particles coming from the core are produced not only at larger impact parameters (and hence more frequently) but also at larger pseudorapidities (hence more forward) as illustrated in Fig. 2. This modified version of EPOS LHC will be called EPOS QGP in the following.
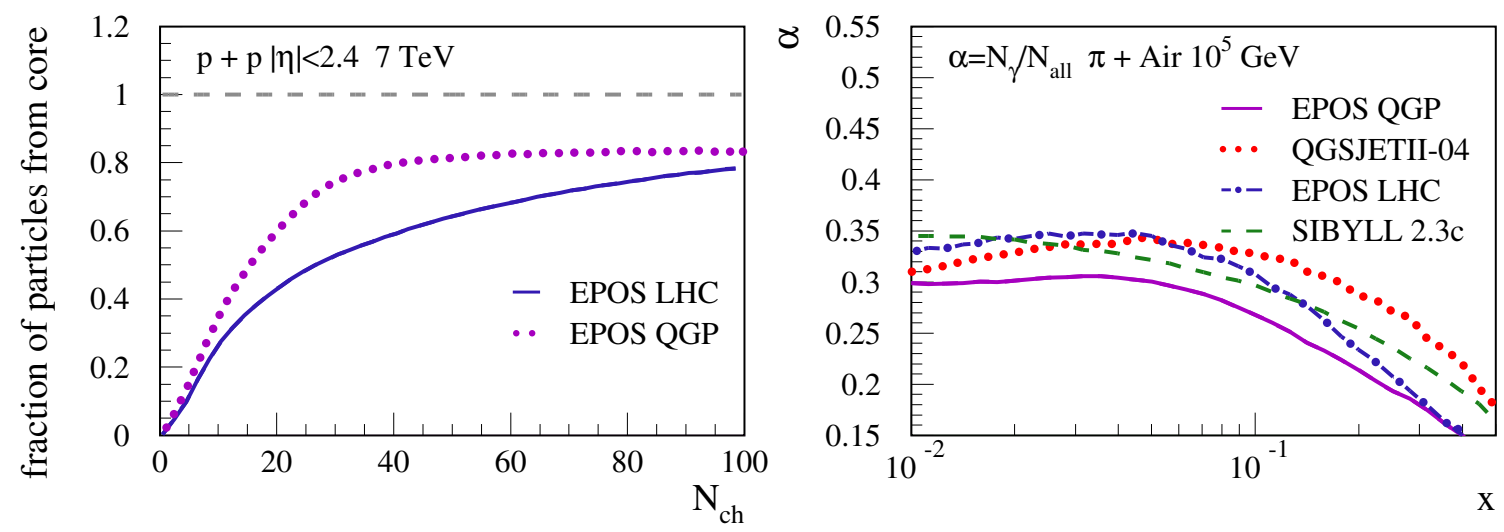

Figure 2: Left side: fraction of particles produced by the core hadronization in a pp collision at $7 \mathrm{TeV}$ as a function of central multiplicity for EPOS LHC (full line) and EPOS QGP (dashed line). Right side: evolution of the ratio between the number of photons over all particles as a function of the momentum fraction $x=E / E_{\max }$ of the produced particles in a $\pi$ Air interaction at $10^{5} \mathrm{GeV}$ lab energy (typical for air showers).

\section{Collective hadronization and air shower physics}

The dominant mechanism for the production of muons in air showers is via the decay of light charged mesons. The vast majority of mesons are produced at the end of the hadron cascade after typically five to ten generations of hadronic interactions (depending on the energy and zenith angle of the cosmic ray). The energy carried by neutral pions, however, is directly fed to the 
electromagnetic shower component and is not available for further production of more mesons and subsequently muons. Thus, the energy carried by hadrons that are not neutral pions is typically able to produce more hadrons and ultimately muons in following interactions and decays. As explained in $[12,19]$, the ratio of the average electromagnetic to average hadronic energy, called $\alpha$, and its dependence on center-of-mass energy, is related to the muon abundance in air showers: if this energy ratio is smaller (larger), less (more) energy is available for the production of muons at the end of the hadronic cascade and ultimately more (less) muons are produced. In fact, it can even be demonstrated in the simple Matthews-Heitler model [20] that the exponent $\beta$ of the energy dependence of the muon production is directly related to $\alpha$ as $\beta=1+\ln (1-\alpha) / \ln \left(N_{\text {all }}\right)$, where $N_{\text {all }}$ is the total multiplicity.

Since in a collective hadronization (or statistical model) the production of particles with higher mass (in particular with strange quarks) [18] is not suppressed as in a string hadronization, the fraction of secondary pions in the dense core is reduced because many other more massive hadrons and resonances are produced. This leads to a lower ratio of the electromagnetic to hadronic energy density in particles produced from the core. Accordingly, this effect can be seen in the momentumfraction-dependent ratio of the average electromagnetic to all particles density $\alpha$ shown in Fig. 2 (right). In EPOS QGP, where the core fraction is increased compared to EPOS LHC, $\alpha$ is reduced by about $15 \%$ is the energy range most relevant for the air shower development (here in $\pi$ Air interactions at $10^{5} \mathrm{GeV}$ lab energy). As a consequence, such reduction of $\alpha$ due to more collectivity in secondary particle production should increase the slope $\beta$ of the energy dependence of the muon

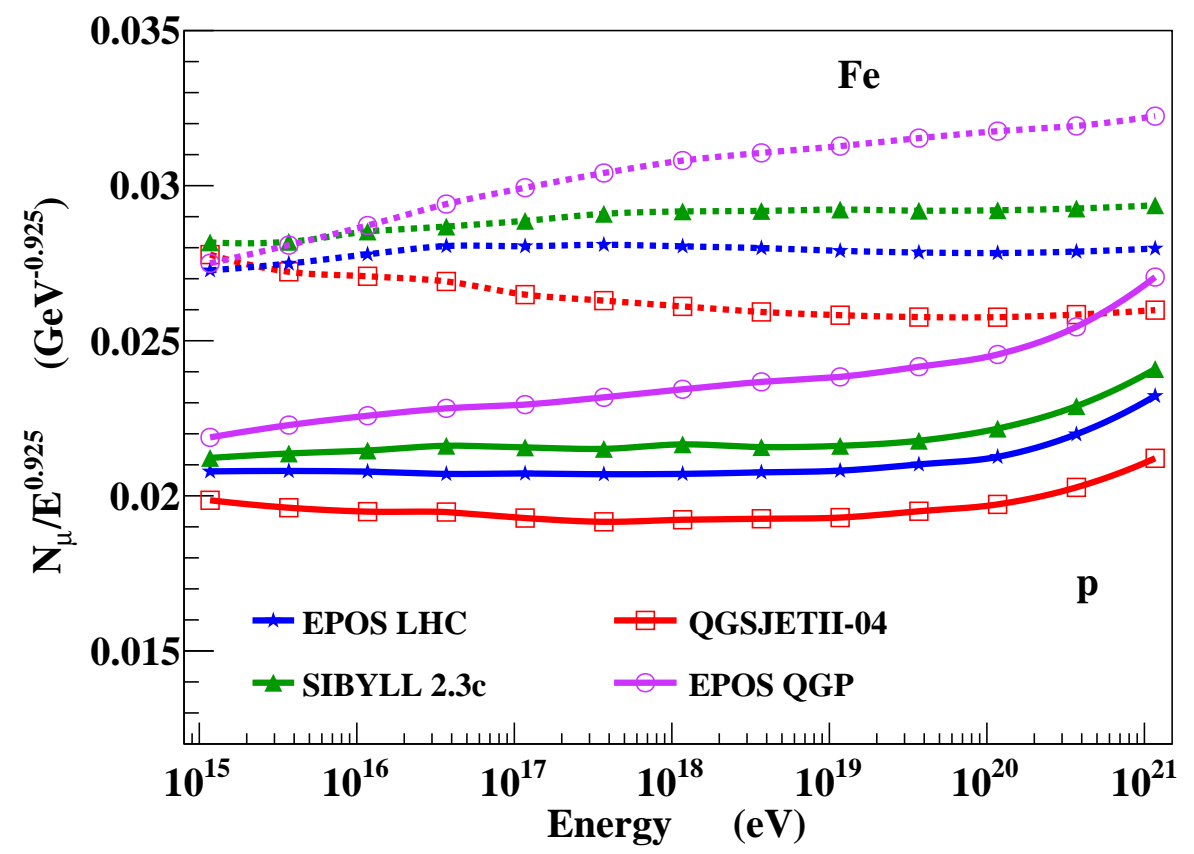

Figure 3: Mean number of muons at the ground divided by the primary energy to the power 0.925 for proton and iron induced showers as a function of the primary energy. Predictions of different high-energy hadronic interaction models: full lines for proton and dashed lines for iron with full stars for EPOS LHC, open circles for EPOS QGP, open squares for QGSJETII-04, and full triangles for the results of Sibyll 2.3c. 
production in air showers.

\section{Results}

As expected, we can see in Fig. 3 that the slope of muon production as a function of the primary energy using EPOS QGP is larger by 1 to 2\% than the one from EPOS LHC (and the other models). This is about half of what would be necessary to reproduce the data compiled in [11]. More detailed studies show that the muon energy spectra are significantly changed towards more muons around $\sim 10 \mathrm{GeV}$ because of the much higher number of strange particles (kaons) produced in the shower. Furthermore, the muon production depth appears to be shallower than the one from Epos LHC.

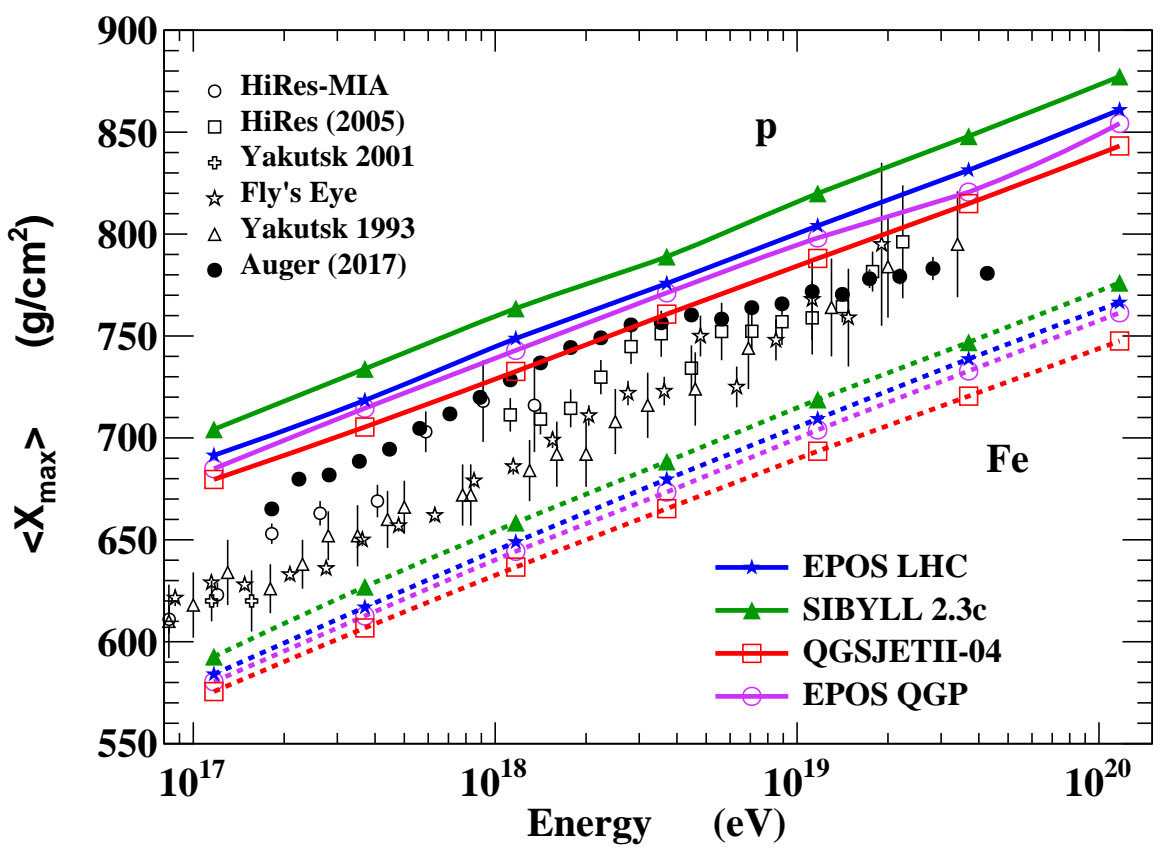

Figure 4: $\left\langle X_{\max }\right\rangle$ for proton and iron induced showers as a function of the primary energy. Predictions of different high energy hadronic interaction models are presented with full lines for proton and dashed lines for iron, with full triangles for Sibyll 2.3c, open circles for EPOS QGP, open squares for QGSJETII-04, full stars for EPOS LHC. Refs. to the data can be found in [21] and [22].

Interestingly, the $\left\langle X_{\max }\right\rangle$ is reduced by a few $\mathrm{g} / \mathrm{cm}^{2}$ (see Fig. 4), but this is not enough to change the interpretation of the cosmic ray composition significantly. In particular, the elongation rate remains the same. As a consequence, the correlation between $\left\langle X_{\max }\right\rangle$ and the number of muons measured by the Pierre Auger Observatory [23] is reproduced better - with about 1 sigma deviation instead of 2 .

\section{Summary}

The improved description of collective hadronization and, in particular, the fact that the core is produced earlier than predicted by EPOS LHC has very important consequences for the muon 
production in air showers. The effect of QGP using the standard EPOS LHC was shown not to be significant. This is since the QGP was produced only for very high-multiplicity events and at mid-rapidity, which are both rare and not important for air shower development. Other studies using a QGP or alternative hadronization as a possible new source of muons were typically based on changes under extreme conditions too $[24,13]$ or with extreme consequences not observed at the LHC [14]. As shown here, according to the most recent LHC results, the collective hadronization starts at a much lower multiplicity and as a consequence affects larger rapidities (lower particle densities than foreseen). In that case, more particles coming from the hadronization of a QGP play a significant role in the air shower development. The production of the QGP is increasing with energy (since the multiplicity increases), and as a consequence, the slope of the energy dependence of the muon production also increases with the primary energy and is closer to the one observed by the WHISP working group [11] in the data, without changing the $\left\langle X_{\max }^{\mu}\right\rangle$ too much. A stronger effect is observed in case of a nuclear projectile, which can create a collective phase with a non-zero chemical potential that can lead to an even stronger increase of the production of non-electromagnetic secondary particles [16]. The combination of a mild increase like observed with EPOS QGP with a proton primary with an even stronger effect for a heavier projectile may be the complete solution of the muon deficit in air shower simulations or at least part of it.

The planned proton+oxygen and oxygen-oxygen collisions at the LHC [25] are very important to test the predictions of EPOS QGP with a collision system that is very close to the common pionair system in an air shower. To that end, the forward rapidity distribution should be measured and $\alpha$, the ratio of average electromagnetic to average hadronic energy [26].

\section{References}

[1] T. Pierog, Air Shower Simulation with a New Generation of post-LHC Hadronic Interaction Models in CORSIKA in proceedings of 35th ICRC, Bexco, Busan, Korea, PoS ( ICRC2017) 1100 (2018).

[2] K.H. Kampert, M. Unger, Astropart.Phys. 35, 660 (2012), arXiv:1201.0018 [astro-ph].

[3] K. Werner, B. Guiot, I. Karpenko, T. Pierog (2013), arXiv:1312.1233 [astro-ph].

[4] T. Pierog, I. Karpenko, J.M. Katzy, E. Yatsenko, K. Werner, Phys. Rev. C 92, 034906 (2015), arXiv:1306.0121.

[5] K. Werner, I. Karpenko, T. Pierog, M. Bleicher, K. Mikhailov, Phys. Rev. C 82, 044904 (2010), arXiv:1004.0805 [astro-ph].

[6] K. Werner, F.M. Liu, T. Pierog, Phys. Rev. C 74, 044902 (2006), arXiv:hep-ph/0506232.

[7] J. Adam et al. (ALICE), Nature Phys. (2017), arXiv:1606.07424 [astro-ph].

[8] J. Pan (ALICE), J. Phys. Conf. Ser. 832, 012044 (2017).

[9] V. Khachatryan et al. (CMS), Phys. Lett. B 742, 200 (2015), arXiv:1409.3392 [astro-ph].

[10] K. Werner, J. Phys. Conf. Ser. 636, 012006 (2015).

[11] H. Dembinski, et al. (EAS-MSU, KASCADE-Grande, IceCube, NEVOD-DECOR, Pierre Auger, SUGAR, Telescope Array, Yakutsk EAS), EPJ Web Conf. 210, 02004 (2019), arXiv:1902.08124 [astro-ph]. 
[12] S. Baur, H. Dembinski, T. Pierog, R. Ulrich, K. Werner, To be published in Phys. Rev. D (2019) arXiv:1902.09265 [astro-ph].

[13] J.F. Soriano, L.A. Anchordoqui, T.C. Paul, T.J. Weiler, PoS ICRC2017, 342 (2018), arXiv:1811.07728 [astro-ph].

[14] J. Alvarez-Muniz, L. Cazon, R. Conceição, J.D. de Deus, C. Pajares, M. Pimenta (2012), arXiv:1209.6474 [astro-ph].

[15] G.R. Farrar, J.D. Allen, EPJ Web Conf. 5307007 (2013), arXiv:1307.2322 [astro-ph].

[16] L.A. Anchordoqui, H. Goldberg, T.K. Weiler, Phys. Rev. D 95, 063005 (2017), arXiv:1612.07328 [astro-ph].

[17] H.J. Drescher, M. Hladik, S. Ostapchenko, T. Pierog, K. Werner, Phys. Rept. 350, 93 (2001), arXiv:hep-ph/0007198 [astro-ph].

[18] K. Werner, et al., EPJ Web Conf. 17, 10900 (2018), arXiv:1812.06330 [astro-ph].

[19] T. Pierog, K. Werner, Phys. Rev. Lett., 171101 (2008), arXiv:astro-ph/0611311.

[20] J. Matthews, Astropart. Phys. 22, 387 (2005).

[21] J. Blümer, R. Engel, J.R. Hörandel, Prog. Part. Nucl. Phys. 63293 (2009), arXiv:0904.0725 [astro-ph].

[22] A. Aab et al., (Pierre Auger), Phys. Rev. D 90122005 (2014), arXiv:1409.4809 [astro-ph].

[23] A. Aab et al., (Pierre Auger), Nucl. Instrum. Meth. A 798172 (2015), doi:10.1016/j.nima.2015.06.058, [arXiv:1502.01323 [astro-ph.IM]].

[24] D. LaHurd, C.E. Covault, JCAP 11, 007 (2018), arXiv:1707.01563 [astro-ph].

[25] Z. Citron et al., Future physics opportunities for high-density QCD at the LHC with heavy-ion and proton beams, Report CERN-LPCC-2018-07 (2018), arXiv:1812.06772 [hep-ph].

[26] H.D. Dembinski, T. Pierog, R. Ulrich, Future proton-oxygen beam collisions at the LHC for air shower physics, in proceedings of 36th ICRC, Madison, Wisconsin, USA, POS ( ICRC2019) 235 (2019). 